\title{
Bulk Viscous Cosmology: Unified Dark Matter
}

\author{
Xu Dou and Xin-He Meng \\ Department of Physics, Nankai University, Tianjin 300071, China \\ Correspondence should be addressed to Xu Dou, dowxdou@gmail.com
}

Received 30 June 2010; Accepted 14 August 2010

Academic Editor: Wing Huen Ip

Copyright ( $\odot 2011$ X. Dou and X.-H. Meng. This is an open access article distributed under the Creative Commons Attribution License, which permits unrestricted use, distribution, and reproduction in any medium, provided the original work is properly cited.

\begin{abstract}
The bulk viscosity is introduced to model the current observational cosmos and the unified dark matter (dark matter and dark energy). The viscous unified model assumes that the universe is filled with a single nonperfect fluid with a bulk viscosity. We review the general framework for the viscous cosmology model building finding that the Hubble parameter has possessed a direct connection with the bulk viscosity coefficient. For the concrete form of the bulk viscosity, the Hubble parameter which has the scaling relation with the redshift can be obtained generally. We discuss two viscosity models and the cosmological evolution to which they lead in detail. Using SNe Ia data, CMB shift, as well as BAO observations, the viscosity model parameters can be fitted very well. We also briefly review the fitting method in this paper.
\end{abstract}

\section{Introduction}

Both the dark matter problem and the cosmic acceleration problem challenge physicists' understanding of the universe. In the standard $\Lambda \mathrm{CDM}$ model, two mixed fluids dark matter and dark energy fluid, are assumed. These two fluids influence the cosmic evolution separately. However, present gravitational probe does not have the ability to differentiate these two fluids. This is the dark degeneracy problem $[1,2]$. It is reasonable to model dark matter and dark energy with single fluid or single-field assumption. Some unified models have been proposed to detect the possibility of this unified assumption, like unified dark fluid model [3-7], which assumes the single-fluid equation of state; Chaplygin gas model and generalized Chaplygin gas model [8-12], which discuss the cosmology consequences of an exotic equation of state; scalar field method [13-15].

The introduction of viscosity into cosmology has been investigated from different viewpoints $[16,17]$. There are some recent developments like dark energy model [18-21], the cosmic singularity [22]. In this paper, we give a brief introduction to unify dark matter and dark energy with viscosity medium. In such models, the universe is assumed to be filled with viscous single fluid [23-29]. The cosmic density is not separated as dark energy part and dark matter part.
The bulk viscosity contributes to the cosmic pressure and plays the role of accelerating the universe. After considering the bulk viscosity, the cosmic pressure can be written as

$$
p=(\gamma-1) \rho-3 \zeta H,
$$

where $\gamma$ parameterizes the equation of state. Generally the form of bulk viscosity is chosen as a time-dependent function. In [30-35], a density-dependent viscosity $\zeta=$ $\alpha \rho^{m}$ coefficient is investigated extensively. For modeling the unified dark matter and dark energy, it is often assumed that the parameter $\gamma=1$, that the pressure of the viscosity fluid is zero, and the viscosity term contributes an effective pressure. There arise some problems here. From the observational results [36], the cosmic density is nearly equals to the cosmic pressure. In the viscosity model, the viscosity term dominates the cosmic pressure and surpasses the pressure contributions from other cosmic matter constitutions, which contradicts the traditional fluid theory. The works in $[37,38]$ propose nonstandard interaction mechanism to solve this problem. Obviously, it is important to build solid foundation for the research of the viscous cosmology.

Equation of state $w<-1$ lies in the phantom region. It is shown that cosmology models with such equation of state possess the so-called the future singularity that is called 
the Big Rip [39]. The larger viscosity model parameter space can help to solve the cosmic singularity problem and produce different kinds of evolution modes of the future universe; for more details [28].

The rest of this paper is organized as follows. In Section 2, general framework of the viscosity model will be reviewed. In Section 3, we discuss the modeling of the unified model with viscosity. In this section, two concrete models are analyzed. In Section 4, data fitting method is introduced briefly.

\section{General Framework}

We consider the standard Friedmann-Robertson-Walker metric

$$
\mathrm{d} s^{2}=-\mathrm{d} t^{2}+a(t)^{2}\left(\frac{\mathrm{d} r^{2}}{1-k r^{2}}+r^{2} \mathrm{~d} \Omega^{2}\right) .
$$

For the sake of simplicity, we choose the flat geometry $k=$ 0 , which is also favored by the update result of the cosmic background radiation measurement.

The general stress-energy-momentum tensor is

$$
T_{\mu \nu}=(\rho+p) U_{\mu} U_{\nu}+p g_{\mu \nu}-\zeta \theta h_{\mu \nu},
$$

where $\zeta$ is the bulk viscosity. The expansion factor $\theta$ is defined by $\theta=U_{; \mu}^{\mu}=3(\dot{a} / a)$, and the projection tensor $h_{\mu \nu} \equiv g_{\mu \nu}+U_{\mu} U_{\nu}$. In the comoving coordinates, the four velocity $U^{\mu}=(1,0,0,0)$. We do not specify the concrete form of $\zeta$ in this section. Generally speaking, $\zeta$ is a quantity evolving with time $t$ or the scale factor $a(t)$. We will see below that nontrivial and more complicated $\zeta$ can produce different results especially useful for the late universe modeling.

From the usual Einstein equation

$$
G_{\mu \nu}=R_{\mu \nu}-\frac{1}{2} R g_{\mu \nu}=8 \pi G T_{\mu \nu},
$$

we obtain the two equations which we call the modified Friedmann equations:

$$
\begin{gathered}
\frac{\dot{a}^{2}}{a^{2}}=\frac{8 \pi G}{3} \rho, \\
\frac{\ddot{a}}{a}=-\frac{4 \pi G}{3}(\rho+3 \tilde{p}),
\end{gathered}
$$

where $\tilde{p}$ is an effective pressure and $\tilde{p}=p-\zeta \theta$.

The covariant conservation equation $T_{; \mu}^{0 \mu}=0$ yields

$$
\dot{\rho}+(\rho+\tilde{p}) \theta=0 .
$$

The existence of a bulk viscosity contributes a modification to the pressure $p$, thus we see that the Friedmann equation and the covariant conservation equation are invariant under the transformation

$$
p \longrightarrow \tilde{p}=p-\zeta \theta .
$$

The covariant energy conservation equation becomes

$$
\dot{\rho}+(\rho-\zeta \theta) \theta=0 .
$$

We define the dimensionless Hubble parameter here as

$$
h^{2}=\frac{H^{2}}{H_{0}^{2}}=\frac{\rho}{\rho_{\mathrm{cr}}},
$$

where $\rho_{\text {cr }}=3 H_{0}^{2} / 8 \pi G$ is the critical density now. Using the dimensionless Hubble parameter, (8) can be transformed as

$$
\frac{1}{H_{0}} \frac{\mathrm{d}\left(h^{2}\right)}{\mathrm{d} t}+3 h^{3}=9 \lambda h^{2},
$$

where the bulk viscosity is redefined as $\lambda=H_{0} \zeta / \rho_{\mathrm{cr}}$. Through the simple relation between scale factor $a(t)$ and the redshift $z$

$$
\mathrm{d} t=\frac{1}{a H} \mathrm{~d} a,
$$

we transform (10) into a differential equation with respect to the scale factor $a(t)$ as follows:

$$
\frac{\mathrm{d} H}{\mathrm{~d} a}+\frac{3}{2 a} H=\frac{3 \zeta}{2 a} .
$$

Solving this equation, we obtain an integral form of $H(a)$ as

$$
H(a)=C_{1} a^{-3 / 2}+\left[\int \frac{3 \zeta}{2 a} \exp \left(\int \frac{3}{2 a} \mathrm{~d} a\right) \mathrm{d} a\right] \exp \left(-\int \frac{3}{2 a} \mathrm{~d} a\right) .
$$

Different forms of viscosity can be used here to make this integral calculable, numerically or exactly.

\section{Unified Single Fluid}

3.1. Redshift-Dependent Model. In [40], the authors assume that the bulk viscosity takes the form of a Hubble parameterdependent function. A redshift-dependent viscosity is proposed in [27]. This bulk viscosity is a combination of a constant and a scaling relation term

$$
9 \lambda=\lambda_{0}+\lambda_{1}(1+z)^{n}
$$

where $n$ is an integer and $\lambda_{0}$ and $\lambda_{1}$ are two constants, which will be fitted from the observational datasets.

After taking account of this ansatz, the integration is easy to work out. We get

$$
\begin{aligned}
h^{2}(z)= & \lambda_{2}^{2}(1+z)^{3}+\frac{2}{3} \lambda_{0} \lambda_{2}(1+z)^{1.5} \\
& -\frac{2 \lambda_{0} \lambda_{1}}{3(2 n-3)}(1+z)^{n}+\frac{\lambda_{1}^{2}}{(2 n-3)^{2}}(1+z)^{2 n} \\
& -\frac{2 \lambda_{1} \lambda_{2}}{2 n-3}(1+z)^{n+1.5}+\frac{\lambda_{0}^{2}}{9} .
\end{aligned}
$$

Since we have assumed the spatial flatness of the universe, the consistency condition requires that $h(0)=1$. Thus this sets a constraint on the model parameters as

$$
\frac{\lambda_{0}}{3}=1-\lambda_{2}+\frac{\lambda_{1}}{2 n-3} \text {. }
$$


We remind the readers that we make the single-fluid assumption above, and we do not concretely specify the constitutions of the cosmic density $\rho$. In this single-fluid model, values of model parameters $\lambda_{0}, \lambda_{1}$, and $\lambda_{2}$ will be fitted, and their meanings are not explained. But when we compare it with two-fluid model, having in mind that the universe is filled with dark matter and dark energy fluid, more constraints can be added. The solution consists of terms with different scaling relation. The first term has the form of $C(1+z)^{3}$, which has the same evolution behavior as that of the cold dark matter. Their simplicity leads us to parameter $\lambda_{2}$ correspond to dark matter ratio $\Omega_{m}$

$$
\lambda_{2}^{2}=\Omega_{m}
$$

This identity can help us utilize more data to constrain the viscosity model. The result is also consistent with that obtained from the standard model $(\Lambda \mathrm{CDM})$. The shift parameter $\mathcal{R}[41,42]$ and the distance parameter $\mathcal{A}$ are defined as

$$
\begin{gathered}
\mathcal{R} \equiv \sqrt{\Omega_{m}} \int_{0}^{z_{*}} \frac{\mathrm{d} z^{\prime}}{h\left(z^{\prime}\right)}, \\
\mathcal{A} \equiv \sqrt{\Omega_{m}} h\left(z_{b}\right)^{-1 / 3}\left(\frac{1}{z_{b}} \int_{0}^{z_{b}} \frac{\mathrm{d} z^{\prime}}{h\left(z^{\prime}\right)}\right)^{2 / 3},
\end{gathered}
$$

respectively. Both of them are dependent on dark matter ratio $\Omega_{m}$, and in the joint statistical analysis, they provide strong constraint on $\Omega_{m}$.

3.2. Effective Equation of State Model. Another viscosity model reviewed here is proposed in [25], where a general form of time-dependent viscosity is discussed:

$$
\zeta=\zeta_{0}+\zeta_{1} \frac{\dot{a}}{a}+\zeta_{2} \frac{\ddot{a}}{\dot{a}}
$$

An interesting feature of this model is its effective equivalence to the following equation of state:

$$
p=(\gamma-1) \rho+p_{0}+w_{H} H+w_{H 2} H^{2}+w_{\mathrm{d} H} \dot{H},
$$

where $p_{0}, w_{H}, w_{H 2}$, and $w_{\mathrm{d} h}$ are free parameters. The corresponding between two groups of coefficients are

$$
\begin{gathered}
w_{H}=-3 \zeta_{0}, \\
w_{H 2}=-3\left(\zeta_{1}+\zeta_{2}\right), \\
w_{\mathrm{d} H}=-3 \zeta_{2} .
\end{gathered}
$$

The parameterized bulk viscosity combines terms related to the "velocity" $\dot{a}$ and "acceleration" $\ddot{a}$, which can be seen to describe the dynamics of the cosmic nonperfect fluid. After eliminating $p$ and $\rho$, a differential equation about the scale factor $a(t)$ can be obtained as

$$
\begin{aligned}
\frac{\ddot{a}}{a}= & \frac{-(3 \gamma-2) / 2-\left(\kappa^{2} / 2\right) w_{H 2}+\left(\kappa^{2}\right) w_{\mathrm{d} H}}{1+\left(\kappa^{2}\right) w_{\mathrm{d} H}}\left(\frac{\dot{a}}{a}\right)^{2} \\
& +\frac{-\left(\kappa^{2}\right) w_{H}}{1+\left(\kappa^{2} / 2\right) w_{\mathrm{d} H}} \frac{\dot{a}}{a}+\frac{-\left(\kappa^{2} / 2\right) p_{0}}{1+\left(\kappa^{2} / 2\right) w_{\mathrm{d} H}} .
\end{aligned}
$$

Another feature of this model is that this differential equation can be solved exactly, and the evolution function of the scale factor $a(t)$ is definite. This evolution function is especially convenient for discussing the cosmic singularity.

With the initial conditions $a\left(t_{0}\right)=a_{0}$ and $\theta\left(t_{0}\right)=\theta_{0}$, when $\tilde{\gamma} \neq 0$, the scale factor can be obtained as

$$
\begin{aligned}
a(t)=a_{0} & \left\{\frac{1}{2}\left(1+\tilde{\gamma} \theta_{0} T-\frac{T}{T_{1}}\right) \exp \left[\frac{t-t_{0}}{2}\left(\frac{1}{T}+\frac{1}{T_{1}}\right)\right]\right. \\
& \left.+\frac{1}{2}\left(1-\tilde{\gamma} \theta_{0} T+\frac{T}{T_{1}}\right) \exp \left[-\frac{t-t_{0}}{2}\left(\frac{1}{T}-\frac{1}{T_{1}}\right)\right]\right\}^{2 / 3 \tilde{\gamma}},
\end{aligned}
$$

where the parameters are redefined as

$$
\begin{gathered}
\tilde{\gamma}=\frac{\gamma+\left(\kappa^{2} / 3\right) w_{H 2}}{1+\left(\kappa^{2} / 2\right) w_{\mathrm{d} H}}, \\
\frac{1}{T_{1}}=\frac{-\left(\kappa^{2} / 2\right) w_{H}}{1+\left(\kappa^{2} / 2\right) w_{\mathrm{d} H}}, \\
\frac{1}{T_{2}^{2}}=\frac{-\left(\kappa^{2} / 2\right) p_{0}}{1+\left(\kappa^{2} / 2\right) w_{\mathrm{d} H}}, \\
\frac{1}{T^{2}}=\frac{1}{T_{1}^{2}}+\frac{6 \tilde{\gamma}}{T_{2}^{2}} .
\end{gathered}
$$

From Friedmann equation, $\rho$ can be written as

$$
\rho(t)=\frac{1}{3 \kappa^{2} \widetilde{\gamma}^{2}}\left[\frac{\left(1+\tilde{\gamma} \theta_{0} T-T / T_{1}\right)\left(1 / T+1 / T_{1}\right) \exp \left(\left(t-t_{0}\right) / T\right)-\left(1-\tilde{\gamma} \theta_{0} T+T / T_{1}\right)\left(1 / T-1 / T_{1}\right)}{\left(1+\tilde{\gamma} \theta_{0} T-T / T_{1}\right) \exp \left(\left(t-t_{0}\right) / T\right)+\left(1-\tilde{\gamma} \theta_{0} T+T / T_{1}\right)}\right]^{2} .
$$

The model parameters leave enough space to produce various evolution behaviors, which can be interpreted in different ways. In this review, we emphasze their power to unify dark energy and dark matter with the single-fluid 
assumption. According to the parameters redefined above and the Friedmann equation, the equation of state can be converted to

$$
p=(\tilde{\gamma}-1) \rho-\frac{2}{\sqrt{3} \kappa T_{1}} \sqrt{\rho}-\frac{2}{\kappa^{2} T_{2}^{2}} .
$$

The case $\tilde{\gamma}=0$ and $T_{1} \rightarrow \infty$ corresponds to the $\Lambda$ CDM. With the aim to unify dark energy and dark matter, the case $\tilde{\gamma}=1$ and $T_{2} \rightarrow \infty$ is especially considered. This case corresponds to a single fluid with constant viscosity. The relation between $p$ and $\rho$ can be obtained from the general equation of state above as follows:

$$
p=-\frac{2}{\sqrt{3} \kappa T_{1}} \sqrt{\rho} .
$$

Therefore, it is straightforward to eliminate $p$ from the covariant energy conservation equation and to work out the solution of $\rho$. Using Friedmann equation, $H(z)$ can be obtained

$$
H(z)=H_{0}\left[\Omega_{\gamma}(1+z)^{3 / 2}+\left(1-\Omega_{\gamma}\right)\right],
$$

where $\Omega_{\gamma}$ is the only one model parameter. Its value can be fitted from $\mathrm{SNe}$ Ia observational data.

\section{Data Fitting}

We review the method to fit the model parameters. More details are illustrated in [43]. The datasets we use are SNe Ia, $\mathrm{BAO}$, and CMB. The 397 Constitution sample [44] combines the Union sample [45] and the low-redshift $(z<0.08)$ sample [46]. The comoving distance $d_{M}$ in FRW coordinate is

$$
d_{M}=\int_{0}^{z} \frac{1}{H\left(z^{\prime}\right)} \mathrm{d} z^{\prime} .
$$

The apparent magnitude which is measured is

$$
m \equiv M+5 \log _{10} D_{L}(z)
$$

where the dimensionless luminosity $D_{L} \equiv H_{0} d_{L}(z)$ and

$$
d_{L}=(1+z) d_{M}(z)
$$

where $M$ is the absolute magnitude which is believed to be constant for all SNe Ia. In the SNe Ia samples, data are given in terms of the distance modulus $\mu_{\mathrm{obs}} \equiv m(z)-M_{\mathrm{obs}}(z)$. The $\chi^{2}$ for this procedure is written as

$$
\chi^{2}=\sum_{i=1}^{n}\left[\frac{\mu_{\mathrm{obs}}\left(z_{i}\right)-\mu_{\mathrm{th}}\left(z_{i} ; c_{\alpha}\right)}{\sigma_{\mathrm{obs}}\left(z_{i}\right)}\right]^{2},
$$

where $\mu_{\text {th }}$ means the distance modulus calculated from model with parameters $c_{\alpha}(\alpha=0,1,2, \ldots)$. Together with the shift parameter $\mathcal{R}$ and the distance $\mathcal{A}$, the total $\chi_{\text {total }}^{2}$ for the joint data analysis is given as

$$
\chi_{\text {total }}^{2}=\chi^{2}+\left(\frac{\mathcal{R}-\mathcal{R}_{\text {obs }}}{\sigma_{\mathcal{R}}}\right)^{2}+\left(\frac{\mathcal{A}-\mathcal{A}_{\text {obs }}}{\sigma_{\mathcal{A}}}\right)^{2} .
$$

For the redshift-dependent model, the relation between distance modulus and redshift is plotted in Figure 1. The model calculated value and the Constitution data are compared in the figure.

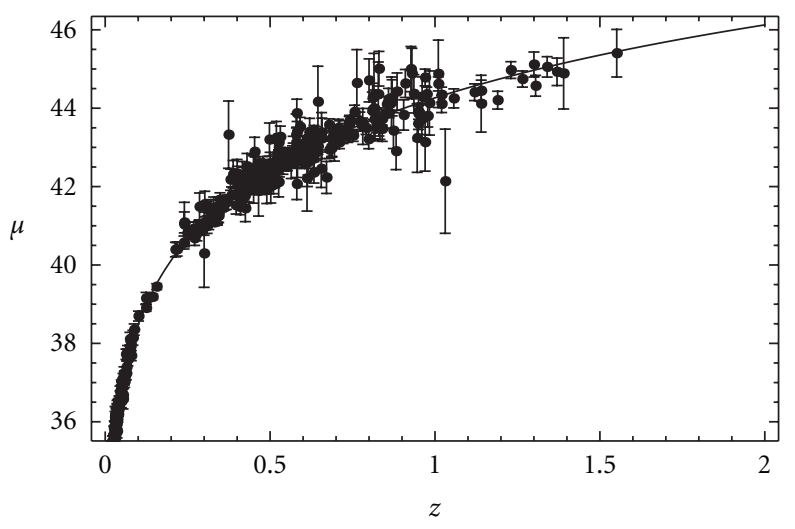

Figure 1: Relation between distance modulus and redshift. The solid line corresponds to the theoretical value calculated from the model concerned. The dots with error bar are the data from the 397 Constitution sample.

\section{Conclusion}

In this review, we discuss three aspects of the viscosity model.

(i) There is general framework for viscosity modeling. General form of Hubble parameter is presented. This general form is convenient for comparing different scale factor- (or redshift-) dependent viscosity models.

(ii) Two kinds of viscosity models are used to model unified models.

(iii) Observation constraint is necessary for model building. We can see that the fitting results are consistent with data. It is prospected that more accurate direct measurements of the Hubble constant will provide a new constraint on cosmological parameters [47].

Especially we focus on its application on modeling the unified dark energy and dark matter.

In the cosmic background level, dynamical analysis can be performed. The statefinder method is useful for discriminating different models [48-52]. Compared with $\Lambda \mathrm{CDM}$ model, evolution of the statefinder of the viscosity model is different and can be discriminated easily; more details can be found in $[28,53]$. More plentiful and accurate data will improve the power of the statefinder method, which will give enough constraint on the late universe model.

We review the viscosity model which is on the level of zero order. The perturbation analysis and to confront the observed large-scale structure are especially useful for the model building and distinguishing. The model predictions need to be consistent with CMB and LSS data. Some works have investigated the perturbation aspects of the viscosity cosmology model $[35,54]$. After choosing properly the corresponding model parameters, the viscosity cosmology model has been shown to have the connection with the Chaplygin gas model. Though the Chaplygin gas model can fit the SNe Ia data well at the zero order, in the perturbation level it is found that the Chaplygin gas model does not behave 
in a satisfactory way. Whether the viscosity cosmology models could behave well needs further investigation.

\section{Acknowledgment}

This work is supported in part by the National Science Foundation of China under No. 11075078.

\section{References}

[1] W. Hu and D. J. Eisenstein, "Structure of structure formation theories," Physical Review D, vol. 59, no. 8, Article ID 083509, 29 pages, 1999.

[2] M. Kunz, "Degeneracy between the dark components resulting from the fact that gravity only measures the total energymomentum tensor," Physical Review D, vol. 80, no. 12, Article ID 123001, 2009.

[3] A. Balbi, M. Bruni, and C. Quercellini, “ $\Lambda \alpha \mathrm{DM}$ : observational constraints on unified dark matter with constant speed of sound," Physical Review D, vol. 76, no. 10, Article ID 103519, 2007.

[4] C. Quercellini, M. Bruni, and A. Balbi, "Affine equation of state from quintessence and k-essence fields," Classical and Quantum Gravity, vol. 24, no. 22, pp. 5413-5425, 2007.

[5] D. Pietrobon, A. Balbi, M. Bruni, and C. Quercellini, "Affine parametrization of the dark sector: constraints from WMAP5 and SDSS," Physical Review D, vol. 78, no. 8, Article ID 083510, 2008.

[6] M. Kunz, A. R. Liddle, D. Parkinson, and C. Gao, "Constraining the dark fluid," Physical Review D, vol. 80, no. 8, Article ID 083533, 2009.

[7] A. Arbey, "Is it possible to consider dark energy and dark matter as a same and unique dark Fluid?" http://arxiv.org/abs/ astro-ph/0506732.

[8] J. D. Barrow, "Graduated inflationary universes," Physics Letters B, vol. 235, no. 1-2, pp. 40-43, 1990.

[9] M. C. Bento, O. Bertolami, and A. A. Sen, "Generalized Chaplygin gas, accelerated expansion, and dark-energy-matter unification," Physical Review D, vol. 66, no. 4, Article ID 043507, 2002.

[10] Z.-H. Zhu, "Generalized Chaplygin gas as a unified scenario of dark matter/energy: observational constraints," Astronomy and Astrophysics, vol. 423, no. 2, pp. 421-426, 2004.

[11] L. Amendola, F. Finelli, C. Burigana, and D. Carturan, "WMAP and the generalized Chaplygin gas," Journal of Cosmology and Astroparticle Physics, vol. 2003, no. 7, article 005, 2003.

[12] X.-H. Meng, M.-G. Hu, and J. Ren, "Cosmology with extended Chaplygin gas media and $w=-1$ crossing," http://arxiv.org/abs/astro-ph/0510357.

[13] S. Nojiri and S. D. Odintsov, "Inhomogeneous equation of state of the universe: phantom era, future singularity, and crossing the phantom barrier," Physical Review D, vol. 72, no. 2, Article ID 023003, 12 pages, 2005.

[14] S. Nojiri and S. D. Odintsov, "Unifying phantom inflation with late-time acceleration: scalar phantom-non-phantom transition model and generalized holographic dark energy," General Relativity and Gravitation, vol. 38, no. 8, pp. 12851304, 2006.

[15] S. Capozziello, S. Nojiri, and S. D. Odintsov, "Unified phantom cosmology: inflation, dark energy and dark matter under the same standard," Physics Letters B, vol. 632, no. 5-6, pp. 597-604, 2006.
[16] $\varnothing$. Grøn, "Viscous inflationary universe models," Astrophysics and Space Science, vol. 173, no. 2, pp. 191-225, 1990.

[17] T. Padmanabhan and S. M. Chitre, "Viscous universes," Physics Letters A, vol. 120, no. 9, pp. 433-436, 1987.

[18] I. Brevik and O. Gorbunova, "Dark energy and viscous cosmology," General Relativity and Gravitation, vol. 37, no. 12, pp. 2039-2045, 2005.

[19] I. Brevik, "Viscous cosmology, entropy, and the CardyVerlinde formula," in Progress in General Relativity and Quantum Cosmology Research, A. Reimer, Ed., vol. 246, p. 165, Nova Science Publishers, 2005.

[20] C.-J. Feng and X.-Z. Li, "Viscous Ricci dark energy," Physics Letters B, vol. 680, no. 4, pp. 355-358, 2009.

[21] A. Avelino and U. Nucamendi, "Exploring a matterdominated model with bulk viscosity to drive the accelerated expansion of the Universe," Journal of Cosmology and Astroparticle Physics, vol. 2010, no. 8, article no. 009, 2010.

[22] I. Brevik and O. Gorbunova, "A brief review of the singularities in $4 \mathrm{D}$ and $5 \mathrm{D}$ viscous cosmologies near the future singularity," http://arxiv.org/abs/0811.1129.

[23] X.-H. Meng, J. Ren, and M.-G. Hu, "Friedmann cosmology with a generalized equation of state and bulk viscosity," Communications in Theoretical Physics, vol. 47, no. 2, pp. 379384, 2007.

[24] J. Ren and X.-H. Meng, "Dark viscous fluid described by a unified equation of state in cosmology," International Journal of Modern Physics D, vol. 16, no. 8, pp. 1341-1348, 2007.

[25] J. Ren and X.-H. Meng, "Cosmological model with viscosity media (dark fluid) described by an effective equation of state," Physics Letters B, vol. 633, no. 1, pp. 1-8, 2006.

[26] J. Ren and X.-H. Meng, "Modified equation of state, scalar field, and bulk viscosity in Friedmann universe," Physics Letters $B$, vol. 636, no. 1, pp. 5-12, 2006.

[27] X.-H. Meng and X. Dou, "Friedmann cosmology with bulk viscosity: a concrete model for dark energy," Communications in Theoretical Physics, vol. 52, no. 2, pp. 377-382, 2009.

[28] X.-H. Meng and X. Dou, "Singularity and entropy of the viscosity dark energy model," http://arxiv.org/abs/0910.2397.

[29] A. Avelino and U. Nucamendi, "Can a matter-dominated model with constant bulk viscosity drive the accelerated expansion of the universe?" Journal of Cosmology and Astroparticle Physics, vol. 2009, no. 4, article 006, 2009.

[30] J. D. Barrow, "The deflationary universe: an instability of the de Sitter universe," Physics Letters B, vol. 180, no. 4, pp. 335339, 1986.

[31] J. D. Barrow, "String-driven inflationary and deflationary cosmological models," Nuclear Physics B, vol. 310, no. 3-4, pp. 743-763, 1988.

[32] J. Barrow, "String-driven inflation," in The Formation and Evolution of Cosmic Strings, G. Gibbons and T. Vasasparti, Eds., pp. 449-464, Cambridge University Press, Cambridge,UK, 1990.

[33] B. Li and J. D. Barrow, "Does bulk viscosity create a viable unified dark matter model?" Physical Review D, vol. 79, no. 10, Article ID 103521, 2009.

[34] R. Colistete Jr., J. C. Fabris, J. Tossa, and W. Zimdahl, "Bulk viscous cosmology," Physical Review D, vol. 76, no. 10, Article ID 103516, 2007.

[35] W. S. Hipólito-Ricaldi, H. E. S. Velten, and W. Zimdahl, "Nonadiabatic dark fluid cosmology," Journal of Cosmology and Astroparticle Physics, vol. 2009, no. 6, article 016, 2009. 
[36] E. Komatsu, K. M. Smith, J. Dunkley et al., "Seven-year Wilkinson Microwave Anisotropy Probe (WMAP) observations: cosmological interpretation," http://arxiv.org/abs/ 1001.4538.

[37] W. Zimdahl, D. J. Schwarz, A. B. Balakin, and D. Pavón, "Cosmic antifriction and accelerated expansion," Physical Review D, vol. 64, no. 6, Article ID 063501, 2001.

[38] A. B. Balakin, D. Pavón, D. J. Schwarz, and W. Zimdahl, "Curvature force and dark energy," New Journal of Physics, vol. 5, pp. 85.1-85.14, 2003.

[39] R. R. Caldwell, M. Kamionkowski, and N. N. Weinberg, "Phantom energy: dark energy with $w<-1$ causes a cosmic doomsday," Physical Review Letters, vol. 91, no. 7, Article ID 071301, 14 pages, 2003.

[40] V. Folomeev and V. Gurovich, "Viscous dark fluid," Physics Letters B, vol. 661, no. 2-3, pp. 75-77, 2008.

[41] J. R. Bond, G. Efstathiou, and M. Tegmark, "Forecasting cosmic parameter errors from microwave background anisotropy experiments," Monthly Notices of the Royal Astronomical Society, vol. 291, no. 3, pp. L33-L41, 1997.

[42] Y. Wang and P. Mukherjee, "Robust dark energy constraints from supernovae, galaxy clustering, and $3 \mathrm{yr}$ Wilkinson microwave anisotropy probe observations," Astrophysical Journal, vol. 650, no. 1, pp. 1-6, 2006.

[43] M. C. Bento, O. Bertolami, N. M. C. Santos, and A. A. Sen, "Supernovae constraints on models of dark energy reexamined," Physical Review D, vol. 71, no. 6, Article ID 063501, 11 pages, 2005.

[44] M. Hicken, W. M. Wood-Vasey, S. Blondin et al., "Improved dark energy constraints from $\sim 100$ new CfA supernova type Ia light curves," Astrophysical Journal, vol. 700, no. 2, pp. 10971140, 2009.

[45] M. Kowalski, D. Rubin, G. Aldering et al., "Improved cosmological constraints from new, old, and combined supernova data sets," Astrophysical Journal, vol. 686, no. 2, pp. 749-778, 2008.

[46] M. Hicken, W. M. Wood-Vasey, S. Blondin et al., "Improved dark energy constraints from $\sim 100$ new CfA supernova type Ia light curves," Astrophysical Journal, vol. 700, no. 2, pp. 10971140, 2009.

[47] L. Greenhill, E. Humphreys, and W. Hu, "Estimation of the hubble constant and constraint on descriptions of dark energy," http://arxiv.org/abs/0902.4255.

[48] V. Sahni, T. D. Saini, A. A. Starobinsky, and U. Alam, "Statefinder-a new geometrical diagnostic of dark energy," JETP Letters, vol. 77, no. 5, pp. 201-206, 2003.

[49] U. Alam, V. Sahni, T. D. Saini, and A. A. Starobinsky, "Exploring the expanding Universe and dark energy using the statefinder diagnostic," Monthly Notices of the Royal Astronomical Society, vol. 344, no. 4, pp. 1057-1074, 2003.

[50] V. Gorini, A. Kamenshchik, and U. Moschella, "Can the Chaplygin gas be a plausible model for dark energy?" Physical Review D, vol. 67, no. 6, Article ID 063509, 2003.

[51] W. Zimdahl and D. Pavón, "Statefinder parameters for interacting dark energy," General Relativity and Gravitation, vol. 36, no. 6, pp. 1483-1491, 2004.

[52] V. Sahni, A. Shafieloo, and A. A. Starobinsky, "Two new diagnostics of dark energy," Physical Review D, vol. 78, no. 10, Article ID 103502, 2008.

[53] M.-G. Hu and X.-H. Meng, "Bulk viscous cosmology: statefinder and entropy," Physics Letters B, vol. 635, no. 4, pp. 186-194, 2006.
[54] J. C. Fabris, S. V. B. Goncalves, and R. de Sa Ribeiro, "Bulk viscosity driving the acceleration of the Universe," General Relativity and Gravitation, vol. 38, no. 3, pp. 495-506, 2006. 

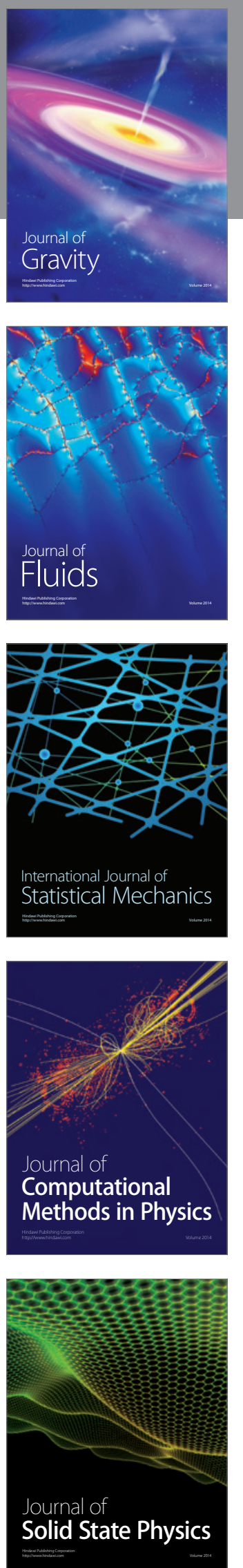

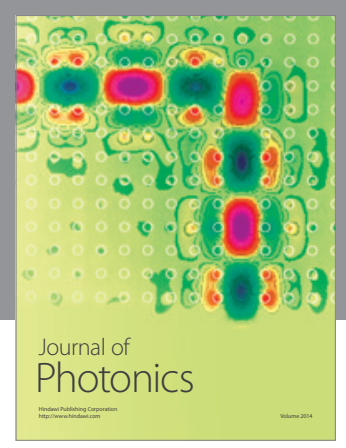

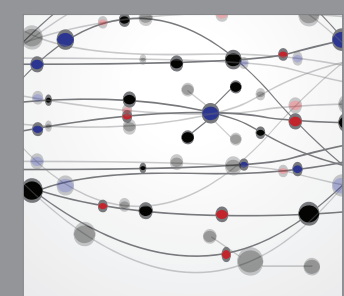

The Scientific World Journal
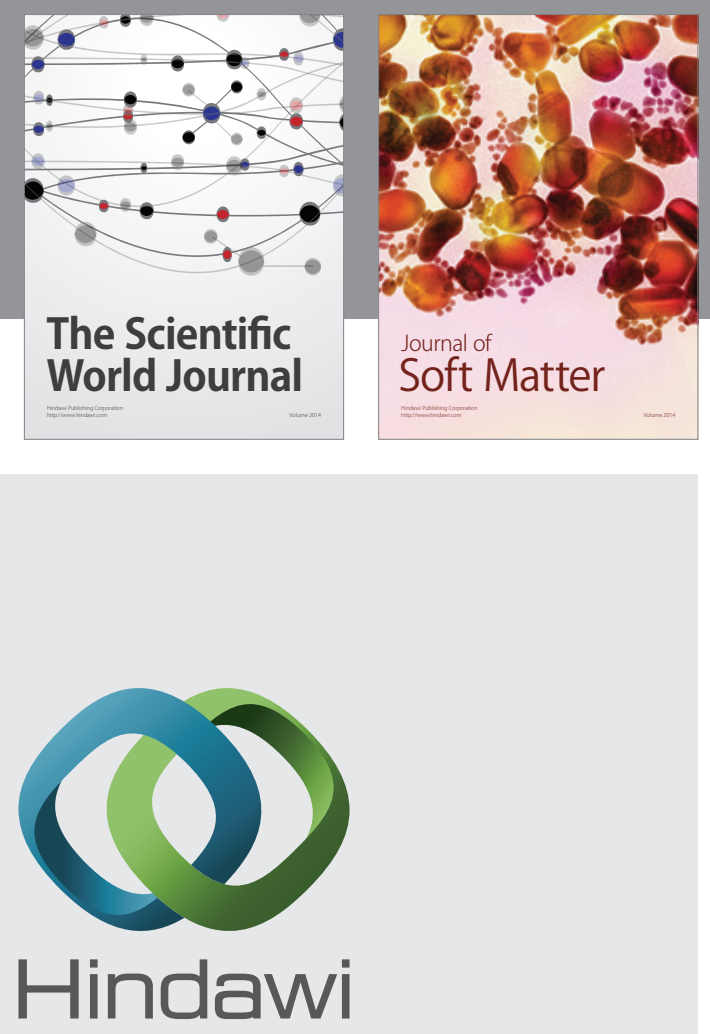

Submit your manuscripts at

http://www.hindawi.com
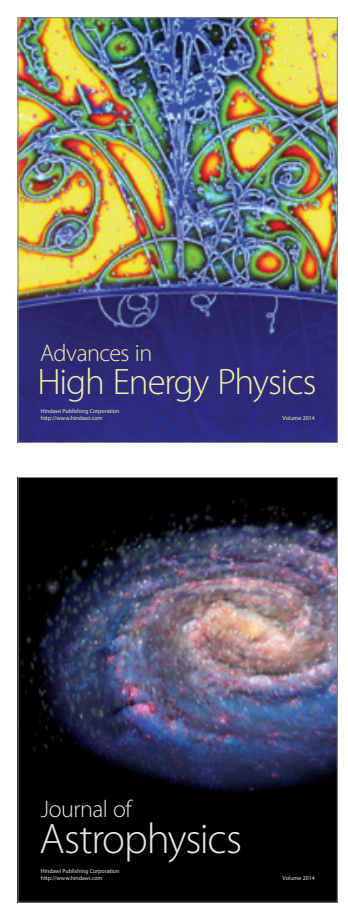
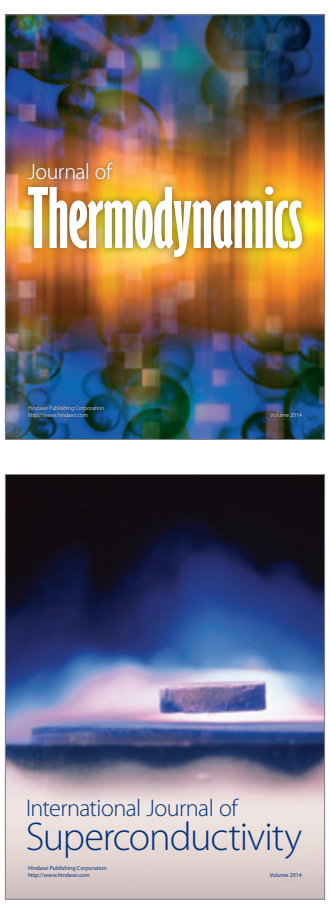
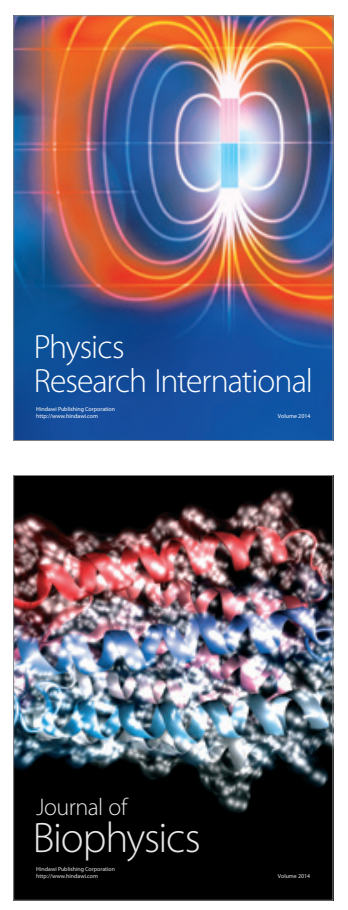
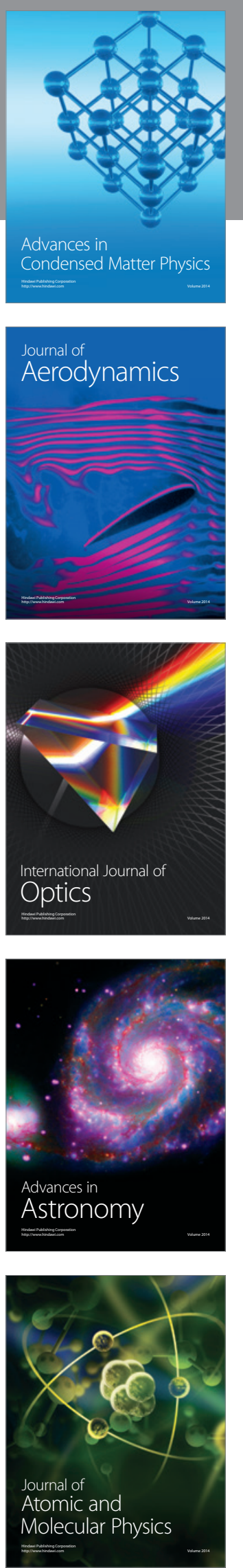